\title{
Combined meta-analysis of systemic effects of allogeneic stem cell transplantation and systemic sclerosis
}

Dmitry N Grigoryev ${ }^{1,2^{*}}$, Jignesh Dalal ${ }^{3}$, Mara L Becker ${ }^{4}$ and Shui Q Ye $e^{1,2}$

\begin{abstract}
Background: Chronic graft-versus-host disease (cGVHD) is a major factor of morbidity and mortality for allogeneic stem cell transplantation (aSCT). The skin and internal organ involvement is the most common systemic complication of CGVHD and closely resembles systemic sclerosis (SSc). Circulating lymphocytes characterize the autoimmune nature of both conditions. Therefore we hypothesized that the common clinical manifestation (systemic organ and skin injury) and the common underlying players (lymphocytes) justify the combined meta-analysis of these diseases.

Results: The aSCT and SSc datasets were uploaded from Gene Expression Omnibus (GEO), a public functional genomics data repository. The available microarray studies of peripheral blood mononuclear cells (PBMCs) and isolated lymphocytes were limited to well established microarray platforms (Affymetrix, Agilent, Canvac, and Illumina) and experimental settings with $\geq 10$ patients per group. The resulting pools of data were merged by unique gene identifier and analyzed by the expression genome-wide association studies (eGWAS) coupled with the subtraction of the cGVHD ${ }^{+}$and $\mathrm{CGVHD}^{-}$ molecular signatures. The eGWAS was applied to 47 and 50 lymphocyte profiles from aSCT and SSC patients, respectively. The identified 35 candidates were represented by 8 known CGVHD genes (including CXCR4, LTBR and PML) and 28 new candidate genes (including SEPX1 and DNJGB1). The further mutual subtraction of CGVHD ${ }^{+}$and $\mathrm{CGVHD}^{-}$candidates and pathway analysis identified a list of 25 genes. Seven of these genes belong to the fibroblast development and function pathway, consisting of the well known CGVHD genes CCND1, JUN, and FOS, and the new molecular targets MMP2, FOSB, TNFAIP8, and DUSP1. These genes become primary candidates for a potential link of systemic effects of CGVHD and SSC.

Conclusions: We designed a new approach for meta-analysis by combining data from different diseases using common clinical manifestation as a linker. This allowed us to power up the insufficient standalone meta-analysis of aSCT microarray studies, by adding SSc samples to the data pool. This new method has successfully identified novel molecular targets for systemic effects of both aSCT and SSc. We believe that this approach is generalizable and can be applied to an array of diseases with common clinical manifestations.
\end{abstract}

Keywords: Meta-analysis, Allogeneic stem cell transplantation, Systemic sclerosis, Microarray, Molecular signature, Gene expression, Public data repository, Peripheral mononuclear cells, Circulating lymphocytes

\footnotetext{
* Correspondence: dgrigoryev@cmh.edu

'Division of Experimental and Translational Genetics, Department of

Pediatrics, Children's Mercy Hospitals and Clinics, Kansas City, MO, USA

${ }^{2}$ Department of Biomedical and Health Informatics, Pediatric Research

Building, Rm\# 4729.02, University of Missouri School of Medicine, 2401

Gillham Road, Kansas City, MO, USA

Full list of author information is available at the end of the article
}

\section{Biomed Central}

(c) 2014 Grigoryev et al.; licensee BioMed Central Ltd. This is an Open Access article distributed under the terms of the Creative Commons Attribution License (http://creativecommons.org/licenses/by/2.0), which permits unrestricted use, distribution, and reproduction in any medium, provided the original work is properly credited. 


\section{Background}

Allogeneic stem cell transplantation (aSCT) has become a curative therapy for increasing numbers of diseases. To date, it is the only successful cellular immunotherapy for high-risk malignancies such as leukemia. In pediatrics, it also offers curative therapy for nonmalignant blood disorders such as thalassemia, immune dysregulation, congenital bone marrow failure syndromes, inborn errors of metabolism and autoimmune conditions [1]. However, $30-70 \%$ of aSCT cases develop cGVHD, which occurs 100 days after transplantation with median time to onset of 4-6 months [2]. The precise mechanisms of cGVHD are unknown and evaluation of global transcriptional changes of reconstituted immune cells from aSCT patients is a feasible approach for studying cGVHD [3]. For instance, the transcript profiling of $\mathrm{CD}^{+}$and $\mathrm{CD}^{+}$lymphocytes from donors for aSCT demonstrated that predictive biomarkers for cGVHD can be detected [4].

Due to the paucity of microarray data for standalone meta-analysis of cGVHD, we used similarities in systemic responses between cGVHD and systemic sclerosis (SSc) to link their microarray datasets $[5,6]$. The similarity in systemic responses between cGVHD and SSc was demonstrated in the experimental settings and was attributed to the excessive activation of $\mathrm{T}$ and $\mathrm{B}$ cells in both conditions [7]. Therefore, we hypothesized that the combined meta-analysis of both diseases would lead to better understanding of systemic effects of circulating lymphocytes in aSCT and SSc.
In this study we combined publicly available datasets for aSCT (with or without cGVHD) and SSc (diffuse and limited) and analyzed them using a meta-analysis technique.

\section{Results and discussion}

A total of 32671 genes were analyzed by eGWAS and 35 genes were identified as associated with both diseases, while 77 genes were associated with SSc only. Genes associated with aSCT alone were not detected (Figure 1 and Additional file 1) due to the low statistical power of cGVHD data. These findings support our approach and provided 35 potential candidates, which otherwise would have been missed by conventional analysis.

Eight genes among these candidates were previously linked to cGVHD (Figure 1), which demonstrated that despite the amplification of cGVHD data with unrelated but similar clinical conditions, we generated a feasible candidate list for $\mathrm{CGVHD}$. The top candidate was lymphotoxin beta receptor or TNFR superfamily, member 3 (LTBR) with $\mathrm{P}=1.73 \times 10^{-13}$ (Figure 1 ). Concordantly, it was recently reported that the expression of $L T B R$ is elevated in patients with $\mathrm{cGVHD}^{+}$as compared to $\mathrm{CGVHD}^{-}$, and that the application of lymphotoxin beta receptorimmunoglobulin fusion protein in mouse model of cGVHD is beneficial $[8,9]$.

Given that eGWAS confirmed the feasibility of combined meta-analysis of two different diseases with common clinical manifestations, we proceeded with the

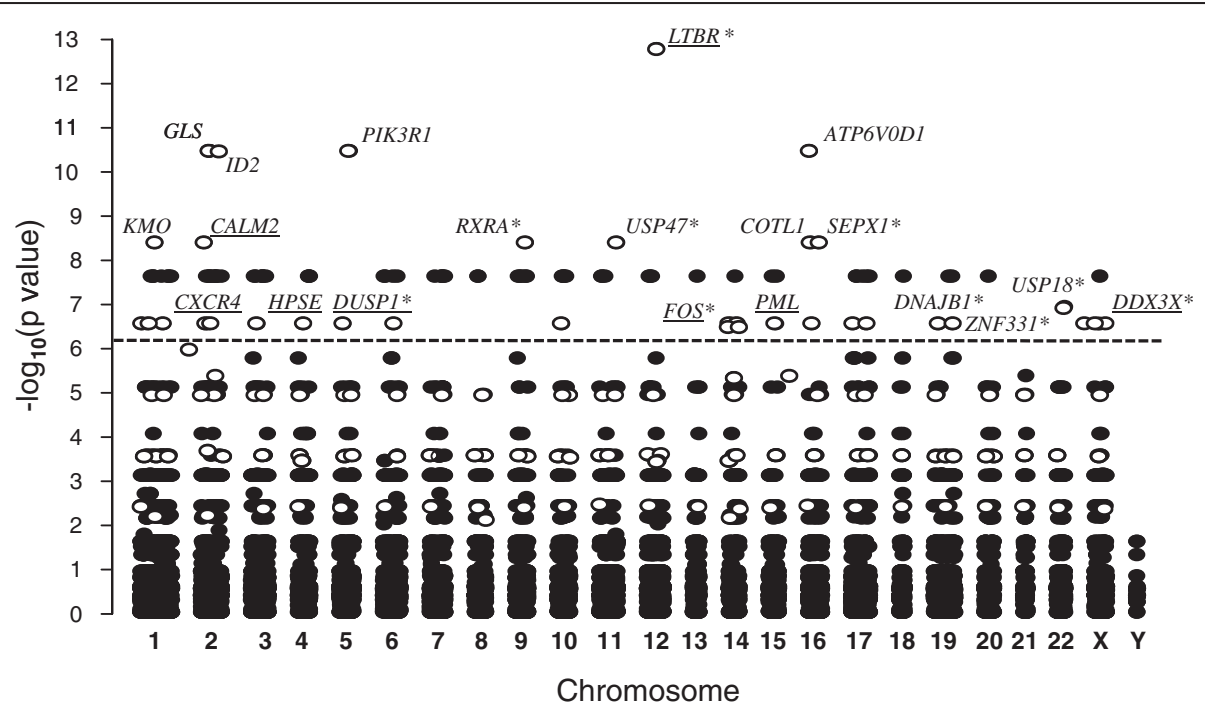

Figure 1 eGWAS for aSCT and SSc using a $x^{2}$ analysis. The significance, P values, of each tested gene is expressed as $-\log _{10}(y$ axis) and plotted against corresponding chromosomal location ( $x$ axis). P values for each gene were calculated for 7 microarray datasets (4 aSCT and 3 SSc) as the likelihood of finding repeated differential expression compared with expected using $X^{2}$ analysis. Out of 32671 genes tested 118 genes demonstrated a significant differential expression, of which 101 had the known chromosomal location. The dotted line indicates the Bonferroni threshold $\left(P=1.53 \times 10^{-6}\right)$. The gene symbols indicate genes that are most significantly associated with both clinical aSCT and SSc. The open circles represent genes that demonstrated significant changes in both diseases and the solid circles represent genes with significant changes only in one of tested diseases. Underscored symbols indicate known cGVHD genes. * - genes that were also detected by subtraction of ranking order gene lists. 
mutual subtraction of molecular signatures of cGVHD and SSc.

We began with stratified analysis of $\mathrm{CD}^{+}$and $\mathrm{CD} 8^{+}$ T-cells from patients with cGVHD and identified 225 $\mathrm{CD}^{+}{ }^{+}$and $155 \mathrm{CD}^{+}$transcripts significantly affected by the new host environment. The analysis of $\mathrm{CD} 4^{+}$and $\mathrm{CD}^{+}$T-cells from patients who underwent aSCT and remained cGVHD ${ }^{-}$one year later identified 800 and 241 transcripts with significant changes, respectively. 84 up- and 141 down-regulated $\mathrm{cGVHD}^{+}$transcripts of $\mathrm{CD} 4^{+}$lymphocytes were cross-referenced against 559 up- and 241 down-regulated aSCT transcripts (Figure 2A). This approach identified 68 up- and 118 down-regulated transcripts that were specific for $\mathrm{CGVHD}^{+}$. In addition, 24 transcripts were oppositely regulated in $\mathrm{cGVHD}^{+}$and $\mathrm{cGVHD}^{-} \mathrm{CD}^{+}$cells: 7 were up-regulated in $\mathrm{cGVHD}^{+}$, but down-regulated in cGVHD ${ }^{-}$cells, and 17 were downregulated in $\mathrm{cGVHD}^{+}$, but up-regulated in $\mathrm{cGVHD}^{-}$cells (Figure 2A), which add up to $210 \mathrm{cGVHD}^{+}$specific transcripts in $\mathrm{CD}^{+}$cells. The similar cross-referencing of $\mathrm{CD}^{+}$T-lymphocytes identified 94 up- and 50 downregulated transcripts that were specific for $\mathrm{CGVHD}^{+}$. In addition, 2 transcripts were oppositely regulated in $\mathrm{cGVHD}^{+}$and $\mathrm{cGVHD}^{-} \mathrm{CD}^{+}$cells: one was up-regulated in $\mathrm{cGVHD}^{+}$, but down-regulated in $\mathrm{cGVHD}^{-}$cells, and another was down-regulated in $\mathrm{CGVHD}^{+}$, but up-regulated in $\mathrm{CGVHD}^{-}$cells (Figure 2B), which were added up to 146 cGVHD $^{+}$specific transcripts in $\mathrm{CD}^{+}$cells.

The comparison of molecular changes of $\mathrm{CD}_{4}^{+}$and $\mathrm{CD}^{+} \mathrm{T}$-lymphocytes 1 year after aSCT was conducted by chi-square test and demonstrated a significant difference $(\mathrm{P}<0.0001)$ in ratios of up- and down-regulated genes between these cell types. In $\mathrm{CD} 4^{+} \mathrm{T}$-cells the molecular signature was shifted towards suppression of gene expression (75 up-regulated genes vs 135 down-regulated genes), while in $\mathrm{CD}^{+} \mathrm{T}$-cells the molecular signature was shifted towards activation (95 up-regulated genes vs 51 down-regulated genes). The overall transcriptional changes were more pronounced in $\mathrm{CD} 4^{+}$cells (210 genes) versus $\mathrm{CD}^{+}$cells (146 genes). These findings suggested that $\mathrm{CD}_{4}^{+}$plays a bigger role in aSCT than $\mathrm{CD} 8^{+}$.

The cross-referencing transcripts associated with cGVHD against transcripts associated with limited or diffuse SSc identified 6 up-regulated genes (Figure 3A) and 19 down-regulated genes (Figure 3B) that were common to both diseases, of which 4 genes were involved in limited SSc and 21 were associated with diffuse SSc.

The significance of co-expression of these 25 genes was evaluated by Student's T-test. The contribution of $\mathrm{CD} 4^{+}$lymphocytes to both cGVHD and SSc was 2.55 times higher $(\mathrm{P}=0.033)$ than the contribution of $\mathrm{CD} 8^{+}$ lymphocytes, which confirmed our observation of more pronounced involvement of $\mathrm{CD}_{4}^{+}$. We next tested which type of SSc the molecular signature of cGVHD lymphocytes most resembles. While we were able to demonstrate that the molecular signature of cGVHD lymphocytes resembles diffuse SSc by 2.2 fold more than limited SSc, the t-test of this comparison did not reach significance $(\mathrm{p}=0.124)$.

The greater contribution of $\mathrm{CD} 4^{+}$to cGVHD molecular signature was further confirmed by the pathway analysis. We evaluated the relevance of cGVHD transcripts to known biological processes using the Ingenuity Pathway Analysis (IPA) tool (http://www.ingenuity.com). 163 out of 210 transcripts of $\mathrm{CD} 4^{+}$lymphocytes and 127 out of 146 transcripts of $\mathrm{CD}^{+}$lymphocytes were mapped
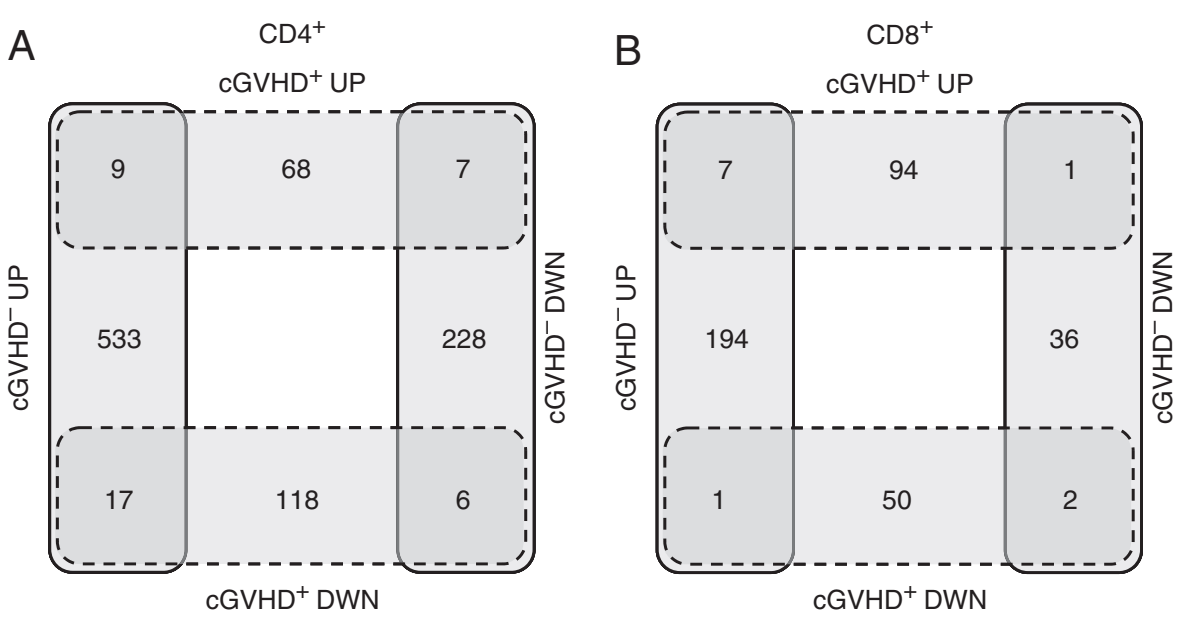

Figure 2 Detection of cGVHD specific transcripts in $\mathrm{CD}^{+}$and $\mathrm{CD}^{+}$lymphocytes. Transcripts that were up- or down- regulated in control population of aSCT patients without CGVHD were subtracted from transcripts that were up- or down- regulated in patients with cGVHD. The subtraction was conducted by cross-referencing of transcriptional changes in $\mathrm{CD}^{+}$(panel $\boldsymbol{A}$ ) or CD8 $8^{+}$(panel $\boldsymbol{B}$ ) lymphocytes, respectively. Transcripts that are common to lymphocytes from control and cGVHD subjects were eliminated from further consideration. 

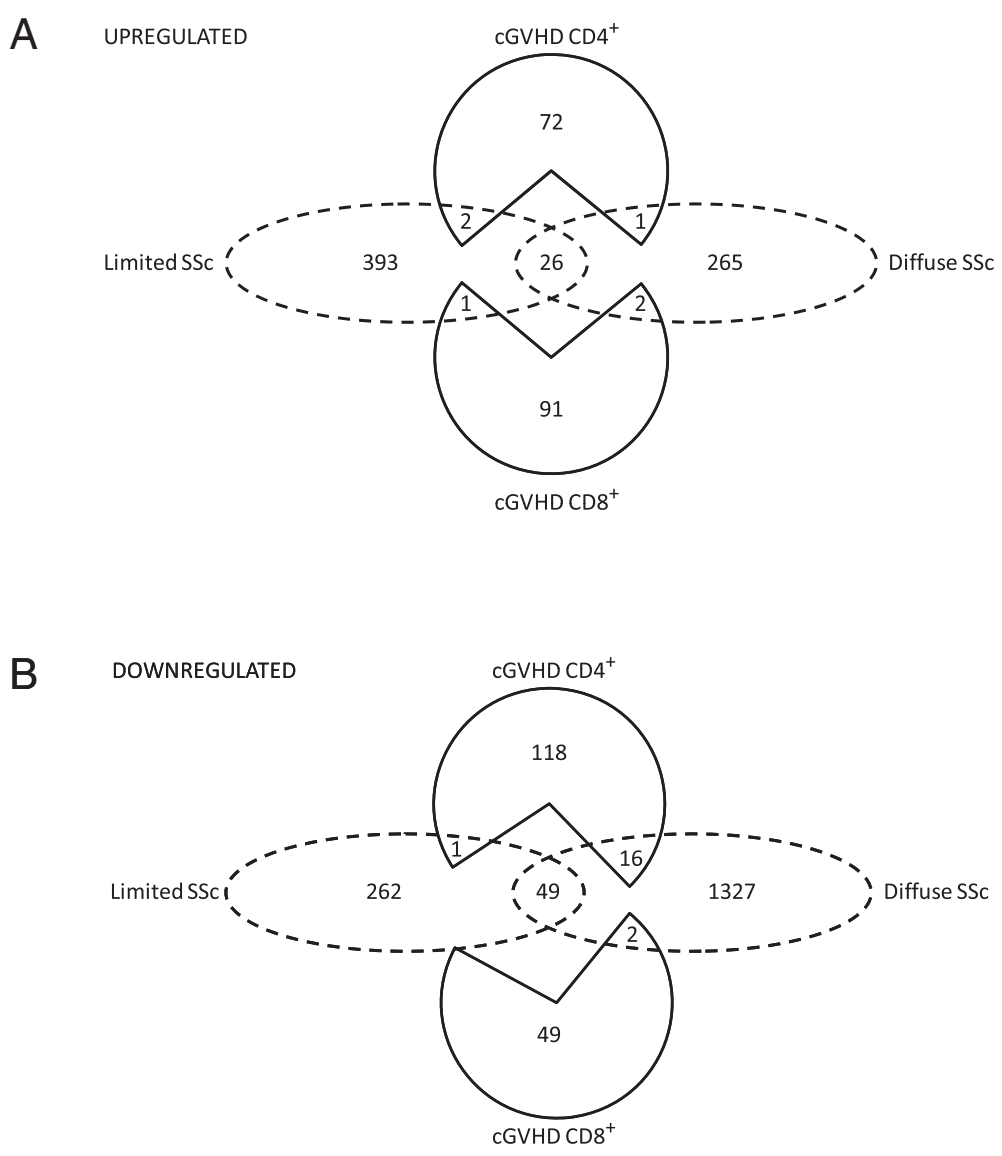

Figure 3 Cross-referencing cGVHD gene candidates with molecular signature of SSc. CD4 ${ }^{+}$(top) and CD8 $8^{+}$(bottom) lymphocytes were cross-referenced against limited SSC or diffuse SSc, respectively. Transcripts that were upregulated (panel $\boldsymbol{A}$ ) or downregulated (panel $\boldsymbol{B}$ ) in both cGVHD and scleroderma patients were considered to be associated with the skin manifestation of cGVHD.

by the software to known genes, while the unmapped transcripts consisted of hypothetical proteins and open reading frames.

The top three bioprocesses identified by IPA for $\mathrm{CD} 4^{+}$ were connective tissue development and function, skeletal and muscular system development and function, and immune cell trafficking. These bioprocesses were built of multiple functional subprocesses, where movement of fibroblasts was the most significant function (Figure 4). None of the top three bioprocesses identified by IPA for $\mathrm{CD}^{+}$contained 5 or more genes, and therefore did not pass filtering criteria.

The top three IPA pathways were overrepresented by fibroblast related genes (7 genes). The immune cell trafficking (5 genes), and muscle cell related functions (4 genes) (Figure 5). While 8 out of 25 candidates that we have detected were reported by the original studies uploaded from GEO, the remaining 17 candidates appeared to be missed as potential cGVHD candidates (Figure 5).

These genes were unreported by the original studies, most likely due to different goals and focuses of the authors. This is an example of how the re-analysis of publicly available data using new approaches will bring to light molecular targets unsuspected by the original studies.

The same group of 25 genes was used for filtering biological networks generated by IPA software. The resulting network represented two major nodes built of $I L 1 B$ and JUN related molecular groups that were cross-linked by FOSB-CCND1 node of fibrotic candidate genes (Figure 6). The existing interactions between these gene candidates suggest their common involvement in the fibrotic response during cGVHD, thus further validating our target selection.

PubMatrix analysis of 25 gene candidates common to cGVHD and SSc identified 7 genes that were previously linked to GVHD (Table 1). The analysis of mutually subtracted $\mathrm{cGVHD}^{+}$and $\mathrm{cGVHD}^{-}$genes identified 6 genes that were previously linked to GVHD, 3 of which were linked to the fibroblast proliferation bioprocess (Table 1). This suggests that our new genes identified by these approaches will be also valid candidates for cGVHD. Moreover, despite the completely different approaches between eGWAS and cross-referencing the molecular 


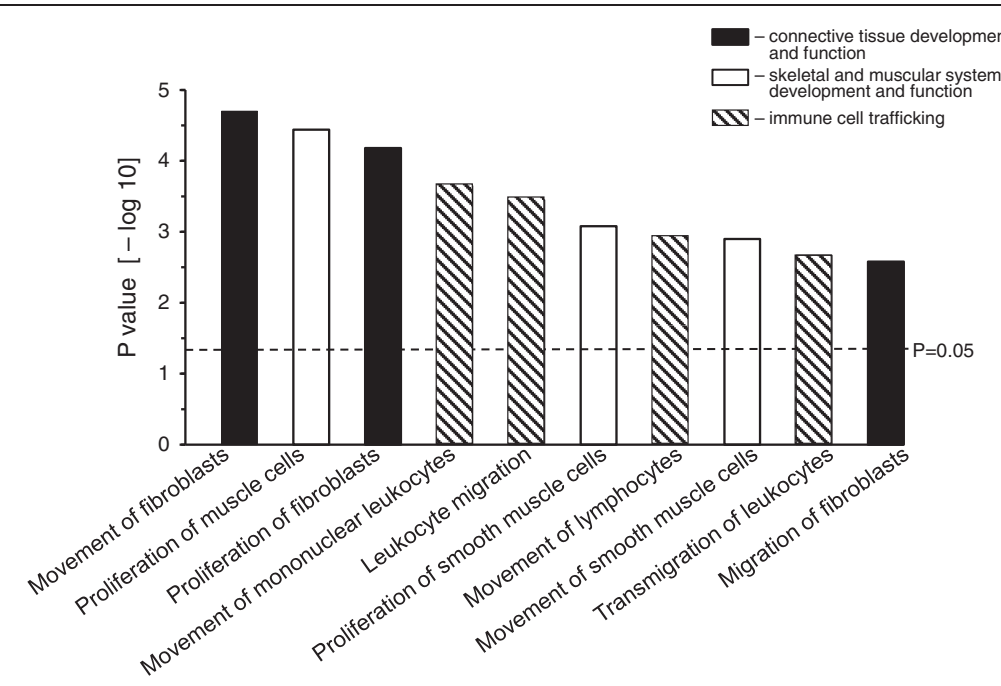

Figure 4 Pathway analysis of cGVHD specific transcripts in CD4 ${ }^{+}$lymphocytes. 210 CGVHD transcripts were analyzed by the Ingenuity Pathway Analysis (IPA) software. IPA mapped 163 of these transcripts to known genes and identified three major processes associated with cGVHD: connective tissue development and function (solid bars), skeletal and muscular system development function (open bars), and immune cell trafficking (hatched bars). The height of each bar represents $-\log _{10} \mathrm{P}$ value $(y$ axis) of corresponding biological function ( $x$ axis). The dashed line indicates the $\mathrm{P}$ value $=0.05\left(-\log _{10} \mathrm{P}=1.301\right)$.

signatures of cGVHD and SSc, 10 genes out of 25 detected by cross-referencing (Figure 5) were also among 35 genes detected by eGWAS (Figure 1).

Of the remaining genes considered new candidates for cGVHD, MMP2, FOSB, TNFAIP8, and DUSP1 were from the fibroblast proliferation pathway and all, but TNFAIP8, were assigned to the same gene-gene interaction network

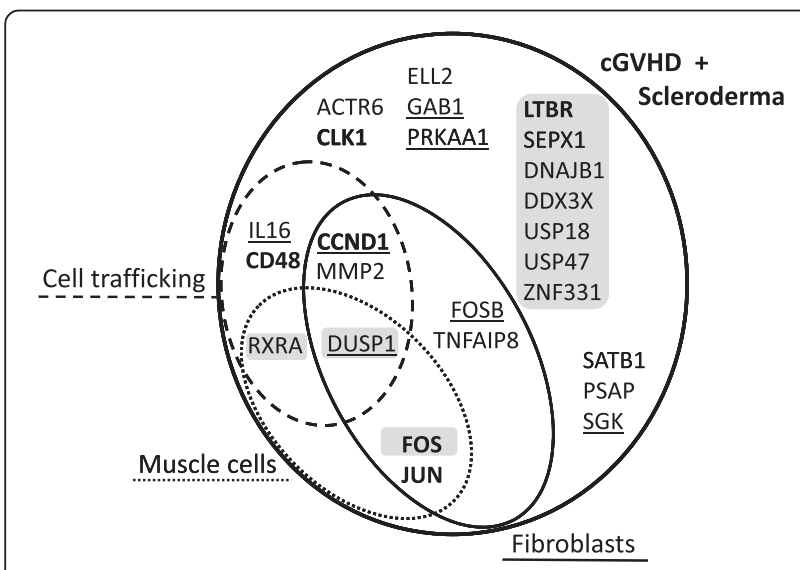

Figure 5 Pathway distribution of cGVHD-SSc gene candidates. The detailed output of pathway analysis was queried against 25 gene candidates (main circle), which are potentially associated with the skin manifestation of CGVHD. Genes were grouped according to involvement in three major pathways (Figure 5): connective tissue development and function (solid oval), skeletal and muscular system development function (dotted oval), and immune cell trafficking (dashed oval). The known cGVHD genes are bolded. The candidates, which were reported in the original aSCT study, are underscored. Genes that were identified by our eGWAS studies (Figures 1) are highlighted by grey boxes.
(Figure 6). Moreover, amongst these genes, DUSP1 was detected by eGWAS studies as well (Figure 1), therefore DUSP1 became our primary candidate.

The expression of DUSP1 in lymphocytes had been detected by many groups (18 citations), however, this gene has not yet been implicated in aSCT or SSc (Table 1). Despite this, the recent studies of circulating cells in peripheral blood of kidney transplant patients identified DUSP1 as a potential biomarker for monitoring renal graft status [10]. We believe that results of our meta-analysis and the reported allograft sensitivity of DUSP1 warrants further studies of this gene in the cGVHD setting.

The other new candidate, $M M P 2$, had the strongest link to aSCT (30 citations) and was detected in scleroderma (2 citations). However it has not yet been linked to cGVHD (Table 1 and Figure 5). In addition to its ability to degrade extracellular matrix proteins, MMP2 can also act on several nonmatrix proteins such as calcitonin generelated peptide, thus promoting vasoconstriction. Moreover, its C-terminal non-catalytic fragment possesses an antiangiogenic property (http://www.uniprot.org). One can speculate that the combination of vasoconstriction and anti-angiogenisity can be contributory to hypoxia and systemic sclerosis. Furthermore, the potential immunogenetic role of MMP2 was observed in a mouse model of heart transplant. It was demonstrated that alloreactivity of T-cells was significantly lower in MMP2-deficient mice compared to their wild type littermates [11]. All these findings make MMP2 an extremely appealing target for cGVHD.

Our final candidate, FOSB, is less known in aSCT (1 citation), but was reported in supplemental materials of 


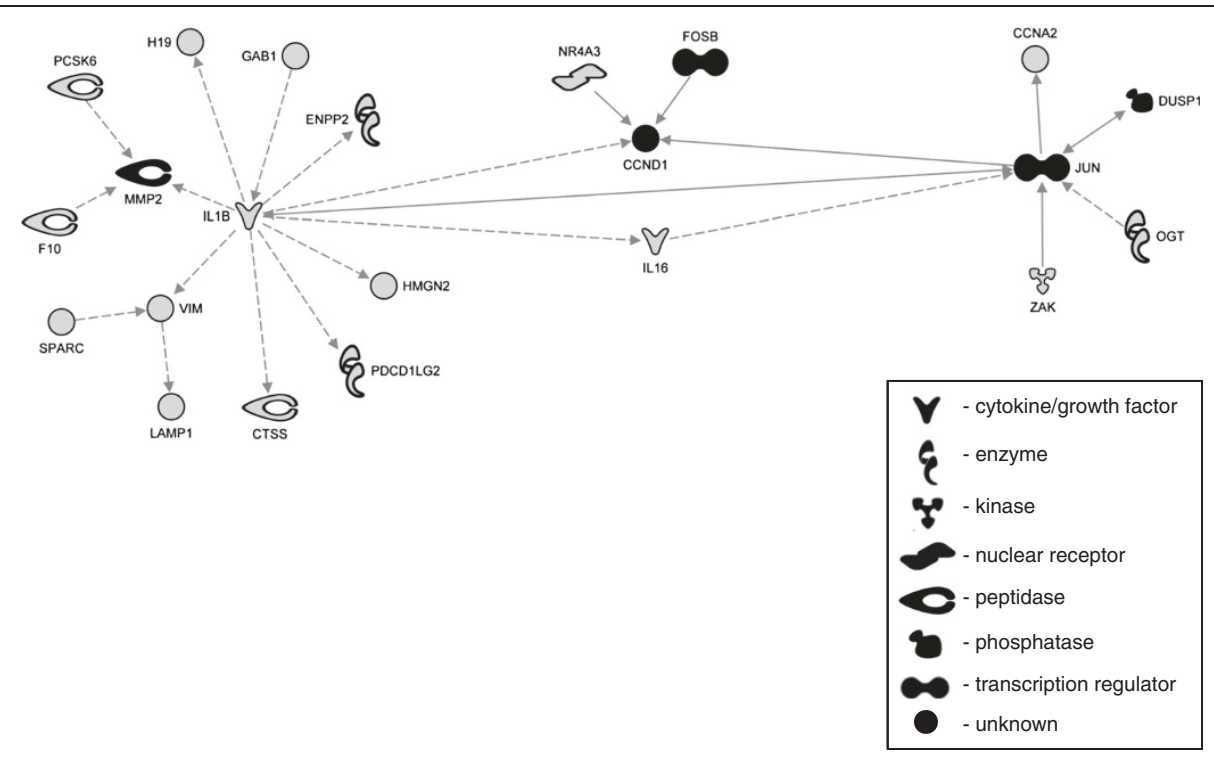

Figure 6 The IPA network analysis of cGVHD-SSc gene candidates. Several networks were generated for 163 cGVHD candidate genes significantly affected by new host environment in $\mathrm{CD}^{+}$lymphocytes. The top network is presented here and is limited to genes with direct relationships with candidates for the skin manifestation of CGVHD. Relationships between genes are represented by solid lines (binding), solid arrows (direct activation), or broken arrows (indirect activation). Arrows point to the element on which an action is performed. Black molecules represent fibroblast related genes. Grey molecules represent other significant cGVHD genes.

original studies submitted to GEO [4]. The gene-gene interaction analysis put this gene into the central cross-linker nodule of two major nodes of cGVHD candidates (Figure 6). The main function of FOSB is believed to enhance binding activity of JUN proteins to DNA. Furthermore, the finding that during induced clonal anergy of $\mathrm{CD}_{4}^{+}$T-cells, JUN and FOS proteins, but not FOSB, reduce their DNA-binding ability [12] makes FOSB an interesting candidate that may prolong the active state of T lymphocytes under suppressive conditions, thus promoting the aSCT complications.

\section{Conclusions}

Presented here data indicate that: 1) for the first time in the field of meta-analysis of microarray data we conducted cross-disease analysis based on common clinical manifestations; 2) $\mathrm{CD} 4^{+} \mathrm{T}$-cells undergo more pronounced changes during cGVHD than their $\mathrm{CD}^{+}$ counterparts and these changes are shifted towards down-regulation; 3) the molecular signature of cGVHD does not demonstrate preferable similarity with either type of systemic sclerosis; 4) the pathway analysis linked expressional changes in lymphocytes from cGVHD patients to fibroblast proliferation; 5) we identified 25 gene candidates common to both cGVHD and SSc, of which primary candidates MMP2, FOSB, and DUSP1are the most appealing for further studies. We believe that our approach of in silico amplification of sparse microarray data by linking molecular signatures of different diseases with the same systemic complications and conducting meta-analysis on such combined dataset is generalizable and capable of detecting new molecular targets.

\section{Methods \\ Data}

The aSCT and SSc datasets were uploaded from gene expression omnibus (GEO, NCBI, http://www.ncbi.nlm. nih.gov/geo). The term "bone marrow transplant" was submitted to the database query windows and this search returned 81 entries (as of December 14, 2012), 23 of which were data series, while the remaining data were either annotation of microarray platforms or individual samples.

The inclusion criteria for retrieved data were the array samples, which must: 1) represent genome wide studies of human peripheral blood mononuclear cells (PBMCs) or isolated lymphocytes; 2 ) be generated with established microarray platforms and the number of interrogated sequences should be $>5000$, thus excluding custom platforms that are pathway oriented and will introduce bias towards given biological process; and 3) the experimental settings must have $\geq 10$ patients per condition.

The studies that satisfied these criteria are listed in Table 2. We utilized data from 5 different experimental settings, which were generated using 4 different microarray platforms. The specific GEO sample IDs (GSM), which were used for this study and a brief description of the experimental settings are listed below:

Baron et al. [4]: GSM103566-67, GSM103610-11, GSM 103616-17, GSM103642-43, GSM103680-81, GSM103684-85, GSM103700-01, GSM103712-13, GSM103716-17, GSM 
Table 1 Gene candidates for skin manifestation of cGVHD

\begin{tabular}{|c|c|c|c|c|c|c|}
\hline Gene & Symbol & Gene & Lympho-cyte & Stem cell transplantation & $\begin{array}{l}\text { Graft versus } \\
\text { host disease }\end{array}$ & $\begin{array}{l}\text { Systemic } \\
\text { sclerosis }\end{array}$ \\
\hline Jun proto-oncogene* & JUN & 13683 & 1546 & 83 & 16 & 33 \\
\hline Lymphotoxin beta receptor & LTBR & 94 & 93 & 6 & 9 & 0 \\
\hline CD48 molecule & CD48 & 136 & 275 & 29 & 4 & 0 \\
\hline Cyclin D1 & CCND1 & 7324 & 424 & 76 & 3 & 1 \\
\hline $\begin{array}{l}\text { FBJ murine osteosarcoma viral oncogene } \\
\text { homolog }\end{array}$ & FOS & 15253 & 836 & 31 & 1 & 10 \\
\hline Interleukin 16 & IL16 & 129 & 242 & 2 & 1 & 1 \\
\hline CDC-like kinase 1 & CLK1 & 27 & 0 & 1 & 1 & 0 \\
\hline Matrix metallopeptidase 2 (gelatinase A) & MMP2 & 906 & 45 & 30 & 0 & 4 \\
\hline $\begin{array}{l}\text { DEAD (Asp-Glu-Ala-Asp) box polypeptide 3, } \\
\text { X-linked }\end{array}$ & DDX3X & 57 & 2 & 2 & 0 & 0 \\
\hline GRB2-associated binding protein 1 & GAB1 & 114 & 23 & 1 & 0 & 0 \\
\hline $\begin{array}{l}\text { FBJ murine osteosarcoma viral oncogene } \\
\text { homolog B }\end{array}$ & FOSB & 522 & 22 & 1 & 0 & 0 \\
\hline $\begin{array}{l}\text { Tumor necrosis factor, alpha-induced } \\
\text { protein } 8\end{array}$ & TNFAIP8 & 14 & 4 & 1 & 0 & 0 \\
\hline Prosaposin & PSAP & 178 & 11 & 0 & 0 & 1 \\
\hline SATB homeobox 1 & SATB1 & 146 & 61 & 0 & 0 & 0 \\
\hline Dual specificity phosphatase 1 & DUSP1 & 392 & 18 & 0 & 0 & 0 \\
\hline Ubiquitin specific peptidase 18 & USP18 & 71 & 7 & 0 & 0 & 0 \\
\hline Dnaj (Hsp40) homolog, subfamily B, member 1 & DNAJB1 & 67 & 7 & 0 & 0 & 0 \\
\hline Elongation factor, RNA polymerase II, 2 & ELL2 & 22 & 6 & 0 & 0 & 0 \\
\hline Serum/glucocorticoid regulated kinase & SGK & 211 & 3 & 0 & 0 & 0 \\
\hline Retinoid $X$ receptor, alpha & RXRA & 77 & 3 & 0 & 0 & 0 \\
\hline Zinc finger protein 331 & ZNF331 & 18 & 2 & 0 & 0 & 0 \\
\hline $\begin{array}{l}\text { Protein kinase, AMP-activated, alpha } 1 \\
\text { catalytic subunit }\end{array}$ & PRKAA1 & 77 & 1 & 0 & 0 & 0 \\
\hline Methionine sulfoxide reductase B1 & SEPX1 & 5 & 1 & 0 & 0 & 0 \\
\hline ARP6 actin-related protein 6 homolog (yeast) & ACTR6 & 1 & 0 & 0 & 0 & 0 \\
\hline Ubiquitin specific peptidase 47 & USP47 & 1 & 0 & 0 & 0 & 0 \\
\hline
\end{tabular}

*Bolded are genes related to fibroblast proliferation.

103720-21, GSM103724-25, and GSM103728-29. Their $\mathrm{CD}^{+} / \mathrm{CD}^{+}$cells were from 12 donors, whose cells did not cause cGVHD in a recipient 1 year after aSCT; GSM103626-27, GSM103630-31, GSM103634-35, GSM103638-39, GSM103650-51, GSM103668-69, GSM 103678-79, GSM103686-87, GSM103689, GSM103696-97, GSM103568-69, GSM103570-71, GSM103573, GSM103
584-85, GSM103592-93, GSM103594-95, GSM103604-05, GSM103704-05, and GSM103707. Their $\mathrm{CD}^{+} / \mathrm{CD}^{+}$cells were from 18 donors, whose cells did cause cGVHD in a recipient 1 year after aSCT (one $\mathrm{CD}^{+}$sample was not reported, see Table 2); GSM103620-21, GSM103640-41, GSM103710-11, GSM103714-15, GSM103718-19, GSM10 3722-23, GSM103726-27, GSM103652-53, GSM103682-83,

Table 2 Summary of microarray data used for meta-analysis

\begin{tabular}{|c|c|c|c|c|c|c|}
\hline Condition & Cell type & Healthy cells & Diseased cells & GEO ID & Microarray platform & Author \\
\hline $\mathrm{aSCT}$ & CD4/CD8 & $12 / 12$ & $10 / 10$ & GSE4624 & Canvac & Baron et al [4]. \\
\hline $\mathrm{aSCT}+\mathrm{cGVHD}$ & CD4/CD8 & $18 / 17$ & $14 / 13$ & GSE4624 & Canvac & Baron et al. [4] \\
\hline Diffuse SSC & PBMCs & 41 & 19 & GSE33463 & Illumina & Cheadle et al. [13] \\
\hline Diffuse SSC & PBMCs & 10 & 10 & GSE22356 & Affymetrix & Risbano et al. [14] \\
\hline Limited SSC & PBMCs & 10 & 21 & GSE19617 & Agilent & Pendergrass et al. [15] \\
\hline
\end{tabular}


and GSM103698-99. Their $\mathrm{CD} 4^{+} / \mathrm{CD}^{+}$cells were from 10 aSCT patients, who remain cGVHD-free 1 year after aSCT; GSM103622-23, GSM103624-25, GSM103628-29, GSM10 3632-33, GSM103636-37, GSM103572, GSM103582-83, GSM103590-91, GSM103618-19, GSM103648-49, GSM10 3666-67, GSM103688, GSM103694-95, GSM103702-03, and GSM103706. Their $\mathrm{CD}^{+} / \mathrm{CD}^{+}$cells were from 14 cGVHD patients, who developed cGVHD 1 year after aSCT (one $\mathrm{CD}^{+}$sample is not reported, see Table 2).

Cheadle et al. [13]: GSM827665-705 - PBMCs were from 41 healthy controls, and GSM827778-96, PBMCs from 19 patients with diffuse SSc.

Risbano et al. [14]: GSM556441-50, PBMCs were from 10 healthy controls, and GSM556413-22, PBMCs from 10 patients with diffuse SSc.

Pendergrass et al. [15]: GSM489236-41, GSM489243, GSM489245-47, PBMCs were from 10 healthy controls, and GSM489194-98, GSM489200-01, GSM489204, GSM 489207-218, PBMCs from 21 patients with limited SSc.

\section{Linking different microarray platforms}

Obtained GSMs were cross-linked based on the GEO provided Minimum Information About a Microarray Experiment (MIAME) data as described previously $[16,17]$. Briefly, the probe IDs across different microarray platforms were linked using the Array Information Library Universal Navigator (AILUN) tool (http://ailun. stanford.edu) and the probes that remained unmatched by AILUN were added to the main AILUN created dataset by matching HUGO-approved gene symbols.

\section{Gene candidate selection}

To estimate differences between groups of samples from aSCT and aSCT + cGVHD patients, and healthy and SSc patients, raw post-quantitation microarray data was median trimmed using minimal trim of 1 highest and 1 lowest expressors. Given the general trend of microarray data series to have low number of biological replicates, the more vigorous trimming was not applied, due to the great reduction of statistical power. The probes without a positive signal throughout all the experiments were then removed, and the remaining data were reanalyzed using SAM 2.0 software [18], applying default settings without application of arbitrary restrictions [19], as described previously [20]. For each gene in every microarray experiment, the $d$ score, which denotes the standardized change in gene expression of diseased lymphocytes versus corresponding healthy lymphocytes, was calculated. Given that the total of 32671 probes were analyzed, the Bonferroni threshold $\left(\mathrm{P}=1.53 \times 10^{-6}\right)$ was used for detecting significance.

For eGWAS analysis, 32671 probes from all experiments were merged into one dataset and significant and not significant changes counted and [4] evaluated by chi-square test as described previously [21]. Genes with an absolute value of $d$ score $\geq 2$ [17] or an absolute value of fold change $\geq 1.2$ [22] between healthy and diseased groups were considered significantly dysregulated.

\section{Molecular signature subtraction}

The gene lists for subtraction analysis were generated using "ranking order" approach. SAM output was ranked according to the fold change and genes from the top quartile were considered significantly affected by a corresponding disease. The subtraction of transcript lists was conducted using VENNY tool (http://bioinfogp.cnb. csic.es/tools/venny/index.html). The significance of association of lymphocyte types $\left(\mathrm{CD}^{+}\right.$or $\left.\mathrm{CD} 8^{+}\right)$in cGVHD samples with the PBMC samples from scleroderma types (diffuse or limited) was assessed using two tailed T-test. Given that scleroderma is represented in the GEO collection by PBMC data only, but knowing that PBMCs are comprised of up to $60 \%$ of $\mathrm{CD}^{+}$and up to $30 \%$ of $\mathrm{CD}^{+}$, we considered it acceptable to directly compare $\mathrm{CD}^{+}$and $\mathrm{CD}^{+}$fractions of cGVHD samples with PBMC samples of SSc patients. Values submitted to the T-test were first normalized to the total number of lymphocyte type or scleroderma type, respectively. Association between molecular signatures with $\mathrm{P}$ value $<0.05$ was considered significant.

\section{Automated literature search}

PubMatrix (multiplex literature mining tool) analysis [23] was conducted as described previously [22]. We restricted our search to human symbols approved by HUGO Gene Nomenclature Committee (HGNC), which were enriched by all aliases and former (discontinued) symbols for selected candidate genes (http://www.genenames.org). The symbols with the hyphen and double names separated by a slash were filtered out. The biomedical literature reference count for a given gene was represented by the highest number among HUGO symbol or its aliases. The genes with number of references $\mathrm{N} \geq 5$ were considered to have established association with cGVHD, $5>\mathrm{N}>0$ were considered novel (have been noticed during cGVHD studies, but were not linked to the disease), and $\mathrm{N}=0$ were considered new.

\section{Pathway analysis}

The pathway analysis, which links the most relevant biological processes to a provided list of candidate genes, was conducted using the Ingenuity Pathways Knowledge Base tool (Ingenuity Systems, Inc., Redwood City, CA.) as described previously [24,25]. Gene symbols from $\mathrm{CD}_{4}^{+}$and $\mathrm{CD}^{+}$significant gene lists were submitted to the IPA and analyzed using "Core" tool. Significance of the identified pathways was tested by the IPA imbedded Fisher Exact test and expressed as p-value [26]. Pathways, that were built of 5 or more genes and had $\mathrm{p}<0.05$ were considered significant. 


\section{Additional file}

\section{Additional file 1: Supplemental Table 1.}

\section{Abbreviations}

AILUN: Array Information Library Universal Navigator; aSCT: Allogeneic stem cell transplantation; cGVHD: Chronic graft-versus-host disease; eGWAS: Expression genome-wide association studies; GEO: Gene expression omnibus; GSM: GEO sample IDs or gene expression series matrix; HGNC: HUGO Gene Nomenclature Committee; IPA: Ingenuity pathways analysis; LTBR: Lymphotoxin beta receptor or TNFR superfamily, member 3: MIAME: Minimum information about a microarray experiment; NCBI: National Center for Biotechnology Information; PBMCs: Peripheral blood mononuclear cells; SSc: Systemic sclerosis.

\section{Competing interest}

The authors declare that they have no competing interests.

\section{Authors' contributions}

DNG, JD and SQY conceived of the study. MLB participated in its design, JD and MLB carried out clinical sample selection from the publicly available data. DNG and SQY did statistical analyses of all data and drafted the manuscript. All authors read and approved the final manuscript.

\section{Acknowledgements}

We thank Dr. Daniel Heruth for his critical reading and editing of our manuscript. This work is in part supported by the start-up fund and William R. Brown/Missouri Endowment of Children's Mercy Hospitals and Clinics, University of Missouri at Kansas City (Ye, S.Q.)

\section{Author details}

${ }^{1}$ Division of Experimental and Translational Genetics, Department of Pediatrics, Children's Mercy Hospitals and Clinics, Kansas City, MO, USA. ${ }^{2}$ Department of Biomedical and Health Informatics, Pediatric Research Building, Rm\# 4729.02, University of Missouri School of Medicine, 2401 Gillham Road, Kansas City, MO, USA. ㄹivision of Hematology Oncology, Department of Pediatrics, Children's Mercy Hospitals and Clinics, Kansas City, MO, USA. ${ }^{4}$ Division of Rheumatology, Department of Pediatrics, Children's Mercy Hospitals and Clinics, Kansas City, MO, USA.

Received: 8 July 2013 Accepted: 6 March 2014

Published: 22 March 2014

\section{References}

1. Rozmus J, Schultz KR: Biomarkers in chronic graft-versus-host disease. Expert Rev Hematol 2011, 4(3):329-342.

2. Lee SJ, Flowers ME: Recognizing and managing chronic graft-versus-host disease. Hematology Am Soc Hematol Educ Program 2008, 1:134-141.

3. Buzzeo MP, Yang J, Casella G, Reddy V: A preliminary gene expression profile of acute graft-versus-host disease. Cell Transplant 2008, 17(5):489-494

4. Baron C, Somogyi R, Greller LD, Rineau V, Wilkinson P, Cho CR, Cameron MJ, Kelvin DJ, Chagnon P, Roy DC, Busque L, Sekaly RP, Perreault C: Prediction of graft-versus-host disease in humans by donor gene-expression profiling. PLoS medicine 2007, 4(1):e23

5. Kitko CL, White ES, Baird K. Fibrotic and sclerotic manifestations of chronic graft-versus-host disease. Biol Blood Marrow Transplant 2012, 18(1 Suppl):S46-52.

6. White JM, Creamer D, du Vivier AW, Pagliuca A, Ho AY, Devereux S, Salisbury JR, Mufti GJ: Sclerodermatous graft-versus-host disease: clinical spectrum and therapeutic challenges. Br J Dermatol 2007, 156(5):1032-1038.

7. Zhang C, Todorov I, Zhang Z, Liu Y, Kandeel F, Forman S, Strober S, Zeng D: Donor CD4+ T and B cells in transplants induce chronic graft-versus-host disease with autoimmune manifestations. Blood 2006, 107(7):2993-3001.

8. Poloni A, Sartini D, Emanuelli M, Trappolini S, Mancini S, Pozzi V, Costantini B, Serrani F, Berardinelli E, Renzi E, Olivieri A, Leoni P: Gene expression profile of cytokines in patients with chronic graft-versus-host disease after allogeneic hematopoietic stem cell transplantation with reduced conditioning Cytokine 2011, 53(3):376-383.
9. Srinivasan M, Flynn R, Price A, Ranger A, Browning JL, Taylor PA, Ritz J, Antin JH, Murphy WJ, Luznik L, Shlomchik MJ, Panoskaltsis-Mortari A, Blazar BR: Donor B-cell alloantibody deposition and germinal center formation are required for the development of murine chronic GVHD and bronchiolitis obliterans. Blood 2012, 119(6):1570-1580.

10. Li L, Khatri P, Sigdel TK, Tran T, Ying L, Vitalone MJ, Chen A, Hsieh S, Dai H, Zhang M, Naesens M, Zarkhin V, Sansanwal P, Chen R, Mindrinos M, Xiao W, Benfield M, Ettenger RB, Dharnidharka V, Mathias R, Portale A, McDonald R, Harmon W, Kershaw D, Vehaskari VM, Kamil E, Baluarte HJ, Warady B, Davis R, Butte AJ, Salvatierra O, Sarwal MM: A peripheral blood diagnostic test for acute rejection in renal transplantation. Am J Transplant 2012, 12(10):2710-2718.

11. Campbell LG, Ramachandran S, Liu W, Shipley JM, Itohara S, Rogers JG, Moazami N, Senior RM, Jaramillo A: Different roles for matrix metalloproteinase- 2 and matrix metalloproteinase- 9 in the pathogenesis of cardiac allograft rejection. Am J Transplant 2005, 5(3):517-528.

12. Heisel O, Keown P: Alterations in transcription factor binding at the IL-2 promoter region in anergized human CD4+ T lymphocytes. Transplantation 2001, 72(8):1416-1422.

13. Cheadle C, Berger AE, Mathai SC, Grigoryev DN, Watkins TN, Sugawara Y, Barkataki S, Fan J, Boorgula M, Hummers L, Zaiman AL, Girgis R, McDevitt MA, Johns RA, Wigley F, Barnes KC, Hassoun PM: Erythroid-specific transcriptional changes in PBMCs from pulmonary hypertension patients. PloS One 2012, 7(4):e34951.

14. Risbano MG, Meadows CA, Coldren CD, Jenkins TJ, Edwards MG, Collier D, Huber W, Mack DG, Fontenot AP, Geraci MW, Bull TM: Altered immune phenotype in peripheral blood cells of patients with scleroderma-associated pulmonary hypertension. Clin Trans/ Sci 2010, 3(5):210-218.

15. Pendergrass SA, Hayes E, Farina G, Lemaire R, Farber HW, Whitfield ML, Lafyatis R: Limited systemic sclerosis patients with pulmonary arterial hypertension show biomarkers of inflammation and vascular injury. Plos One 2010, 5(8):e12106.

16. Chen R, Li L, Butte AJ: AILUN: reannotating gene expression data automatically. Nat Methods 2007, 4(11):879.

17. Grigoryev DN, Cheranova DI, Heruth DP, Huang P, Zhang LQ, Rabb H, Ye SQ: Meta-analysis of molecular response of kidney to ischemia reperfusion injury for the identification of new candidate genes. BMC Nephrol 2013, 14:231.

18. Tusher VG, Tibshirani R, Chu G: Significance analysis of microarrays applied to the ionizing radiation response. Proc Natl Acad Sci U S A 2001, 98(9):5116-5121.

19. Larsson O, Wahlestedt C, Timmons JA: Considerations when using the significance analysis of microarrays (SAM) algorithm. BMC Bioinform 2005, 6:129.

20. Grigoryev DN, Mathai SC, Fisher MR, Girgis RE, Zaiman AL, Housten-Harris T, Cheadle C, Gao L, Hummers LK, Champion HC, Garcia JG, Wigley FM, Tuder RM, Barnes KC, Hassoun PM: Identification of candidate genes in scleroderma-related pulmonary arterial hypertension. Trans/ Res 2008, 151(4):197-207.

21. Kodama K, Horikoshi M, Toda K, Yamada S, Hara K, Irie J, Sirota M, Morgan AA Chen R, Ohtsu H, Maeda S, Kadowaki T, Butte AJ: Expression-based genome-wide association study links the receptor CD44 in adipose tissue with type 2 diabetes. Proc Natl Acad Sci U S A 2012, 109(18):7049-7054.

22. Grigoryev DN, Ma SF, Irizarry RA, Ye SQ, Quackenbush J, Garcia JG: Orthologous gene-expression profiling in multi-species models: search for candidate genes. Genome Biol 2004, 5(5):R34.

23. Becker KG, Hosack DA, Dennis G Jr, Lempicki RA, Bright TJ, Cheadle C, Engel J: PubMatrix: a tool for multiplex literature mining. BMC Bioinform 2003, 4:61.

24. Grigoryev DN, Liu M, Hassoun HT, Cheadle C, Barnes KC, Rabb H: The local and systemic inflammatory transcriptome after acute kidney injury. J Am Soc Nephrol 2008, 19(3):547-558.

25. Hassoun HT, Lie ML, Grigoryev DN, Liu M, Tuder RM, Rabb H: Kidney ischemia-reperfusion injury induces caspase-dependent pulmonary apoptosis. Am J Physiol Renal Physiol 2009, 297(1):F125-137.

26. Jimenez-Marin A, Collado-Romero M, Ramirez-Boo M, Arce C, Garrido JJ: Biological pathway analysis by ArrayUnlock and Ingenuity Pathway Analysis. BMC Proc 2009, 3(Suppl 4):S6.

doi:10.1186/2052-1839-14-7

Cite this article as: Grigoryev et al:: Combined meta-analysis of systemic effects of allogeneic stem cell transplantation and systemic sclerosis. BMC Hematology 2014 14:7. 